\title{
High Energy-Resolution EELS and SXES Studies on Characteristic Chemical Shifts and Charge Transfer in Al-Si-Mn and Zn-Mg-Zr Alloys
}

\author{
S. Koshiya, M. Terauchi, S. Ohhashi and A. P. Tsai
}

Institute of Multidisciplinary Research for Advanced Materials, Tohoku University, 2-1-1 katahira, Aoba-ku, Sendai 980-8577, JAPAN

In recent years, Hume-Rothery mechanism, which predicts a existence of a pseudogap around the Fermi level $\mathrm{E}_{\mathrm{F}}$, is accepted as a major reason for the stabilization of quasicrystals. The presences of pseudogap in quasicrystals were confirmed by X-ray photoemission spectroscopy and electron energyloss spectroscopy (EELS). EELS experiments also pointed out characteristic chemical shifts in Al Lshell excitation spectra of Al-based quasicrystals [1], which suggested a decrease of valence electron charge at Al sites. Recently, a covalent bonding nature in quasicrystals was reported by MEM/Rietveld analysis [2]. Thus, it is interesting to investigate the relation between a chemical shift and bonding nature of the quasicrystals.

In this study, the chemical shift of $\mathrm{Al}_{53} \mathrm{Si}_{27} \mathrm{Mn}_{20}$ alloys [3] and $\mathrm{Zn}-\mathrm{Mg}-\mathrm{Zr}$ alloys [4] were investigated by using EELS and soft-X-ray emission spectroscopy (SXES). For EELS and SXES measurements, a high energy-resolution EELS-TEM [5] and an SXES-TEM [6] were used. Those results of the amount of chemical shifts are compared with those of pure materials or their oxides.

Figure 1(a) shows Al L-shell excitation (EELS) spectra of amorphous (Am), quasicrystalline (QC) and crystalline (Cryst) phases of $\mathrm{Al}_{53} \mathrm{Si}_{27} \mathrm{Mn}_{20}$ alloys. Only the QC phase shows an apparent shift to the larger binding energy side by $0.4 \mathrm{eV}$ compared with that of $\mathrm{Al}$ single crystal. This result should be due to a shift of Al L-level of QC phase to the larger binding energy side (chemical shift). Figure 1(b) show SXES spectra of Al-K $\mathrm{K}_{\alpha}$ and $\mathrm{Si}-\mathrm{K}_{\alpha}$ of Am, QC and Cryst phases. A peak position of each spectrum is assigned as the top of intensity maximum indicated by a vertical line. Al- $\mathrm{K}_{\alpha}$ emission of QC phase clearly shows a shift to the larger energy side by $4 \mathrm{eV}$. This tendency is consistent with that of EELS measurements. Si-K $\mathrm{K}_{\alpha}$ of QC phase also shows a clear shift. However, there was not a chemical shift for Mn- $\mathrm{L}_{\alpha}$. This suggests that the amount of valence electron of $\mathrm{Al}$ and $\mathrm{Si}$ atomic sites decreased, expect Mn sites, in the QC phase [7].

Figure 2(a) shows Zn M-shell excitation (EELS) spectra of Zn-Mg-Zr alloys of primitive icosahedral quasicrystal (P-QC), face-centered icosahedral quasicrystal (F-QC) and a 1/1-approximant (1/1-AP) of F-QC phases. Only P-QC and F-QC alloys show an apparent shift to larger binding energy side compared with pure $\mathrm{Zn}$. The apparent chemical shifts in QC alloys were also observed in $\mathrm{Mg} \mathrm{L}-$ shell excitation spectra (not shown here). Figure 2(b) shows $\mathrm{Zn}-\mathrm{L}_{\alpha}$ emission spectra of the alloys. The $\mathrm{Zn}-\mathrm{L}_{\alpha}$ emission of pure $\mathrm{Zn}$ and $\mathrm{ZnO}$ are also shown for comparison. P-QC and F-QC alloys clearly show a shift to the larger binding energy side by about $2 \mathrm{eV}$ compared with pure Zn. These shifts are comparable to that of $\mathrm{ZnO} .1 / 1-\mathrm{AP}$ does not show any shift within experimental accuracy.

Those results show a presence of characteristic chemical shift not only in Al-based quasicrystalline alloy but also in Zn-based quasicrystalline phase. Therefore, a chemical shift to the larger binding energy side (decrease of valence charges on atomic sites) can be a common characteristic in quasicrystalline states. It strongly suggest that an increase of covalency in quasicrystal states.

Present work was partly supported by Grant-in-Aid for Scientific Research on Priority Areas 'New Materials Science Using Regulated Nano Spaces-Strategy in Ubiquitous Elements' by the Ministry of Education, Culture, Sports, Science and Technology of Japan (No.19051002). 
References

[1] M. Terauchi et al., Philos. Mag., 87 (2009) 2947. [2] K. Kirihara et al., Phys. Rev. B, 64 (2001) 212201. [3] A.P. Tsai et al., Phys. Rev. B, $\underline{49}$ (1994) 3569. [4] S. Ohhashi et al., Acta Mater., 57 (2009) 4727. [5] M. Mukai et al., Microsc. Microanal., 12 (Supp 2) (2006) 1206. [6] M. Terauchi et al., J. Electron Microsc., 59 (2010) 251. [7] S. Koshiya et al., Philos. Mag., accepted.

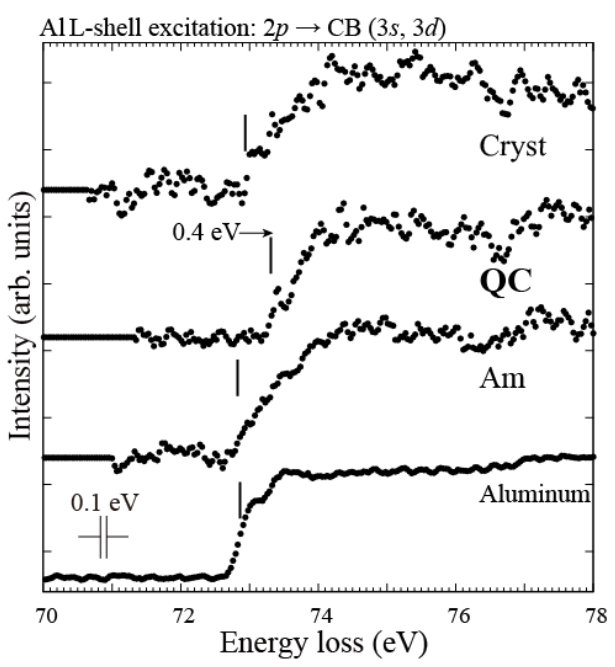

FIG 1(a). Al L-shell excitation (EELS) spectra of Am, QC and Cryst phases of Al-Si-Mn alloy and pure Al.
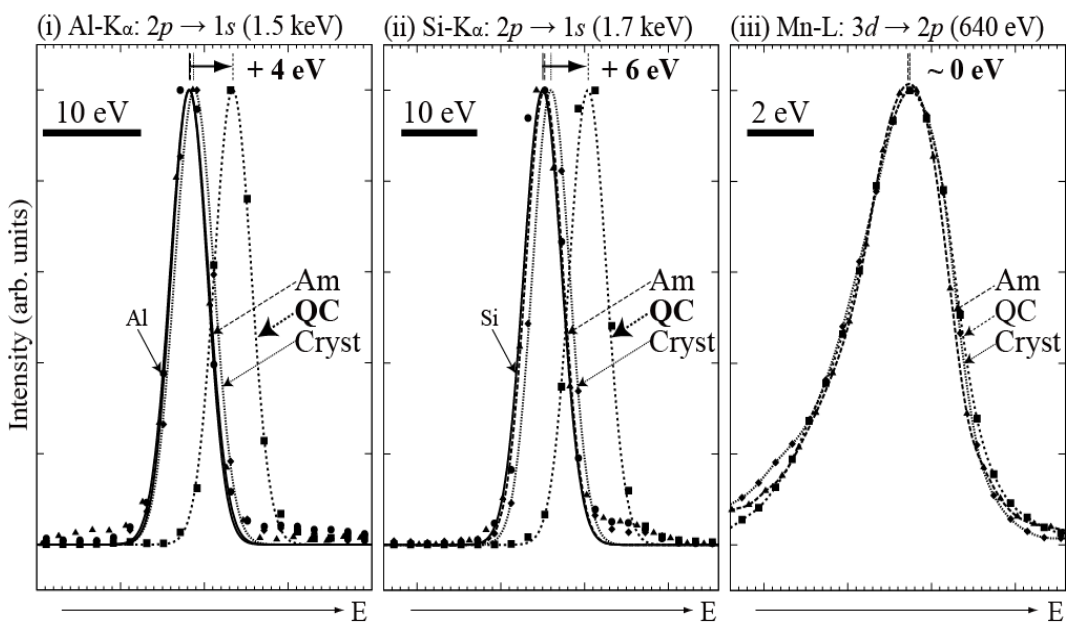

FIG 1(b). Al-K $\mathrm{K}_{\alpha}, \mathrm{Si}-\mathrm{K}_{\alpha}$ and Mn-L emission (SXES) spectra of Am, QC and Cryst phases of Al-Si-Mn alloy.

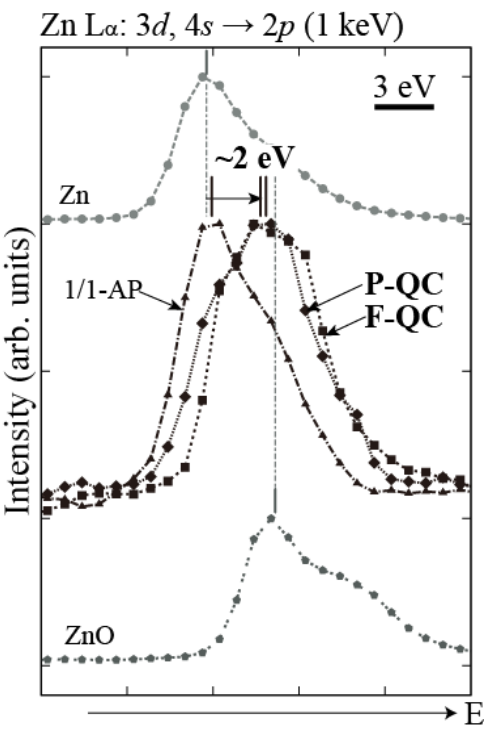

FIG 2(b). Zn $\mathrm{L}_{\alpha}$-emission spectra of P-QC, F-QC and 1/1-AP alloys of Zn-Mg-Zr, pure $\mathrm{Zn}$ and $\mathrm{ZnO}$. 